\title{
Dipeptidyl Peptidase 4 Inhibitors and Venous Thromboembolism Risk in Patients with Type 2 Diabetes: A Meta-analysis of Cardiovascular Outcomes Trials
}

\author{
Li Xin ${ }^{1}$ Shusen Sun ${ }^{2,3,4}$ Jinwen Wang ${ }^{1}$ Wenchao Lu ${ }^{5,6}$ Tiansheng Wang ${ }^{7}$ Huilin Tang ${ }^{8}$
}

\footnotetext{
${ }^{1}$ Department of Clinical Pharmacy, The Second People's Hospital of Yunnan Province, Kunming, Yunnan, China

${ }^{2}$ College of Pharmacy and Health Sciences, Western New England University, Springfield, Massachusetts, United States

${ }^{3}$ Department of Pharmacy, Xiangya Hospital, Central South University, Changsha, Hunan, China

4 The Hunan Institute of Pharmacy Practice and Clinical Research, Changsha, Hunan, China

${ }^{5}$ Department of Pharmacy, Beijing Chaoyang Hospital, Capital

Medical University, Beijing, China

6 Department of Pharmacy Administration and Clinical Pharmacy, School of Pharmaceutical Sciences, Peking University, Beijing, China

7 Department of Epidemiology, Gillings School of Global Public Health, University of North Carolina at Chapel Hill, Chapel Hill, North Carolina, United States

8 Institute for Drug Evaluation, Peking University Health Science Center, Beijing, China
}

Thromb Haemost 2021;121:106-108.

Dipeptidyl peptidase 4 (DPP-4) inhibitors are commonly prescribed second-line oral glucose-lowering drugs in the treatment of type 2 diabetes, which have good safety profiles compared with other antidiabetic drugs, such as low risk of hypoglycemia and having neutral effects on cardiovascular outcomes. One recent pharmacovigilance study using World Health Organization Vigibase found a consistent signal of reporting of venous thromboembolism (VTE) associated with DPP-4 inhibitors (proportional reporting ratio [PRR]: 2; 95\% confidence interval $[\mathrm{Cl}]: 1.7-2.3$ ) especially at the gastrointestinal levels (PRR: 13.4; 95\% CI: 9.2-19.6). ${ }^{1}$ Our study using Food and Drug Administration adverse-event reporting system database found no association between DPP-4 inhibitors and VTE risk but with a moderate signal of VTE at gastrointestinal levels. ${ }^{2}$ However, spontaneous reporting databases have several limitations, such as reporting bias, lack of denominator data, and confounding; this association requires further investigation. Current evidence regarding the effect of DPP-4 inhibitors on VTE risk is very limited, we thus performed a meta-analysis of large cardiovascular outcomes trials (CVOTs) to evaluate the association

received

March 31, 2020

accepted after revision

July 4, 2020

Address for correspondence Huilin Tang, MSc, Institute for Drug Evaluation, Peking University Health Science Center, No 49 North Garden Road, Haidian District, Beijing 100191, China (e-mail: huiltang85@gmail.com).

between DPP-4 inhibitors and VTE risk among the patients with type 2 diabetes.

We systematically searched Pubmed, Embase, and Cochrane Central Register of Controlled Trials (CENTRAL) up to May 1, 2020 to identify the large randomized, double-blind, placebocontrolled CVOTs. Two reviewers independently selected the trials according to the following inclusion criteria: (1) CVOTs involving patients with type 2 diabetes; (2) DPP-4 inhibitors versus placebo; (3) trials reported the events of VTE including pulmonary embolism and gastrointestinal VTE. The events of VTE, pulmonary embolism, or gastrointestinal VTE were defined based on the MedDRA ${ }^{1}$ and were retrieved from the results of serious adverse events reported on Clinicaltrials.gov. The risk of bias of included trials was judged as low, high, or unclear according to the Cochrane risk of bias tool. ${ }^{3} \mathrm{~A}$ pooled odds ratio (OR) with $95 \% \mathrm{CI}$ was calculated using a fixed effects model and an $I^{2}$ statistic was used to assess the possible between-study heterogeneity. Furthermore, a meta-regression was performed to test the difference between the DPP-4 inhibitor and a funnel plot as well as the Egger's test was performed to evaluate the publication bias. We considered $p$ -

(c) 2021. Thieme. All rights reserved. Georg Thieme Verlag KG,

Rüdigerstraße 14,

70469 Stuttgart, Germany
DOI https://doi.org/ 10.1055/s-0040-1715444. ISSN 0340-6245. 
value $<0.05$ as statistically significant. The meta-analysis was performed with STATA(Version 14; Stata Corp., College Station, Texas, United States).

Finally, five CVOTs were included in this meta-analysis. ${ }^{4-8}$ The basic characteristics of the included trials are summarized in - Table 1. A total of 47,714 patients with type 2 diabetes and established or at high risk of cardiovascular disease were randomly allocated to the DPP-4 inhibitor group (23,899 patients) and the placebo group (23,815 patients). The median duration of follow-up ranged from 1.8 to 3 years. The mean age, baseline $\mathrm{HbA} 1 \mathrm{c} \%$, and baseline body mass index on included patients were 64.2 years, $7.8 \%$, and $30.5 \mathrm{~kg} / \mathrm{m}^{2}$, respectively. The risk of bias of each trial was judged as low ( - Table 1 ). Results of individual trials as well as meta-analysis are presented in - Fig. 1. For risk of VTE, 79 events in the DPP-4 inhibitor group and 70 events in the placebo group were reported. Meta-analysis of the five trials did not find a significant association between DPP-4 inhibitors and risk of VTE (OR: 1.12; 95\% CI: 0.81-1.55). Furthermore, there was no significant difference between DPP-4 inhibitors $(p=0.50)$. Similarly, no significant difference between DPP-4 inhibitors (31 events) and placebo (27 events) was observed regarding the risk of pulmonary embolism (OR: 1.14; 95\% CI: 0.68-1.90). For risk of gastrointestinal VTE, only two events were reported in patients taking DPP-4 inhibitors and none were reported in the placebo group. There was no significant association between DPP-4 inhibitors and placebo (OR: 2.98 ; 95\% CI: 0.31-28.70). No statistical heterogeneity was observed in the meta-analysis with an $I^{2}$ ranged from 0 to $26.7 \%$. There was no evidence of publication bias in the meta-analysis based on funnel plot (figures not shown) and Egger's test ( $p>0.05)$.

To our knowledge, this is the first meta-analysis of placebo-controlled CVOTs to assess the risk of VTE associated with DPP-4 inhibitors among patients with type 2 diabetes. Some evidence from spontaneous reporting databases indicated that DPP-4 inhibitors were associated with an increased risk of VTE. ${ }^{1,9}$ However, we found no significant difference in the risk of VTE between DPP-4 inhibitors and placebo, which was also consistent with our previous study. ${ }^{2}$ It has been proposed that DPP-4 inhibitors may have protective mechanisms against thrombosis effects by inhibiting platelet aggregation through their inhibitory effect on intracellular free calcium and tyrosine phosphorylation ${ }^{10}$ and improving endothelium-dependent vasodilatation through increasing circulating levels of GLP-1. ${ }^{11} \mathrm{An}$ increase in events of VTE at the gastrointestinal levels was observed in previous studies, ${ }^{1,2}$ while in this study only two events were reported in the DPP-4 inhibitor group, which requires further investigation including their underlying mechanisms.

This study has several strengths including meta-analysis of available strong evidence and only considering randomized placebo-controlled trials that balanced the background therapy as well as other confounders. However, some limitations in this study should be considered. First, the events of VTE were not the predefined outcomes in these trials that the numbers of VTE are likely underestimated as only serious

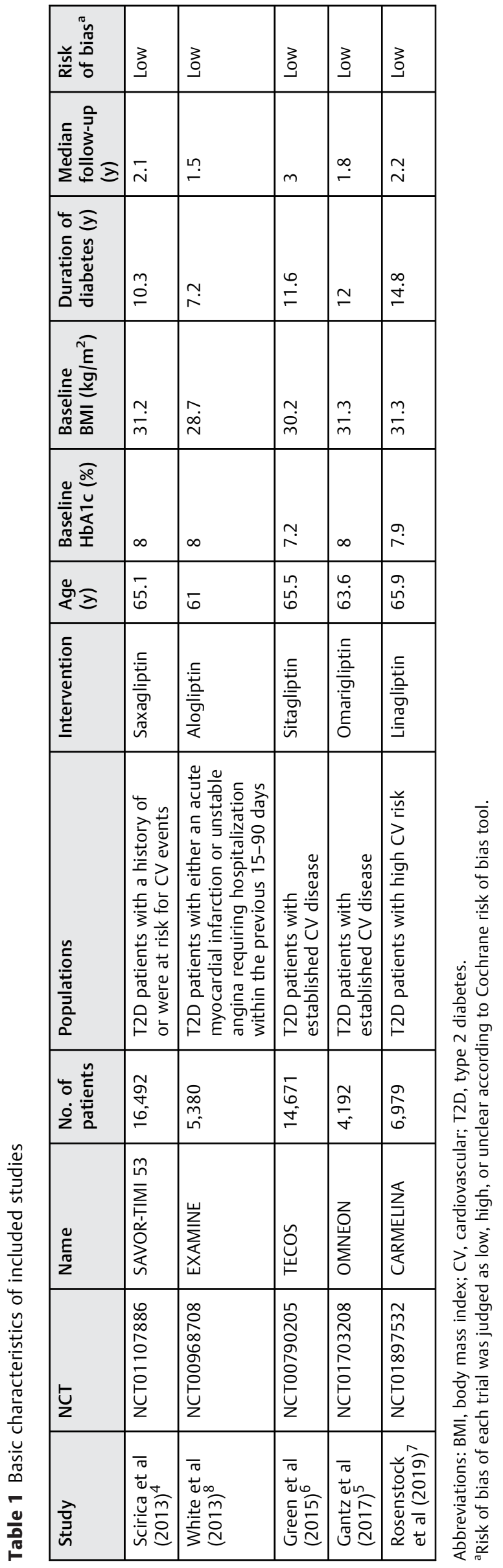




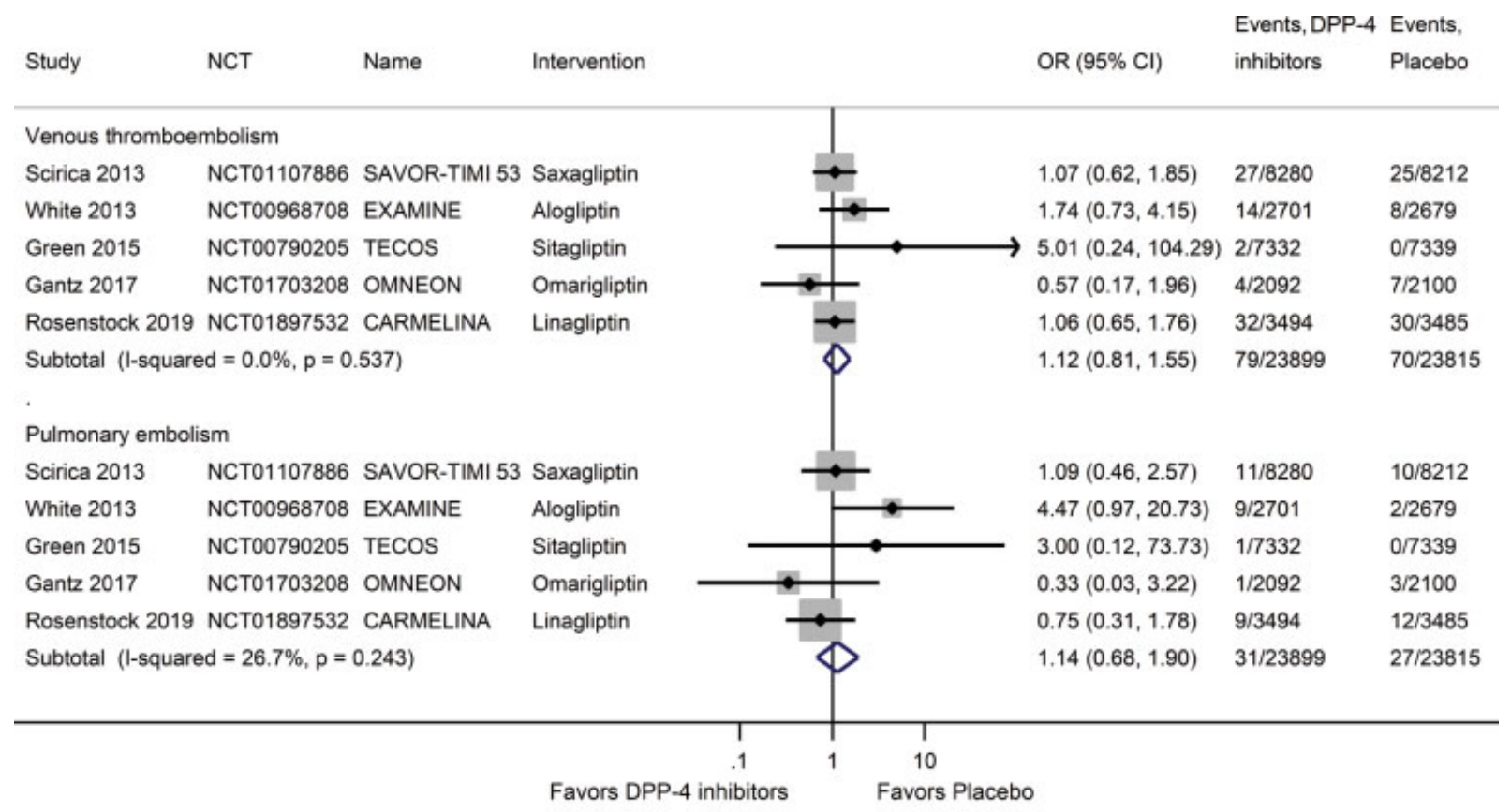

Fig. 1 Meta-analysis of the association between DPP-4 inhibitors and risk of venous thromboembolism and pulmonary embolism.

adverse events of VTE were reported on the Clinicaltrials.gov. Second, given the low incidence of VTE in this study, the study might be underpowered to detect that difference as significant. For example, only two events of VTE were identified at the gastrointestinal levels. Therefore, we cannot fully exclude the increased risk of VTE associated with DPP-4 inhibitors among the patients with type 2 diabetes. Third, due to lack of information regarding the co-medication in individual patients from trials, we cannot evaluate the potential drug-drug interaction in this study.

In conclusion, based on current available evidence from CVOTs, we did not find a significant difference between DPP4 inhibitors and risk of VTE. However, further meta-analyses of individual data from all randomized controlled trials as well as well-designed large prospective observational studies are warranted to confirm our findings.

Funding

None.

Conflict of Interest

None declared.

\section{References}

1 Gouverneur A, Lair A, Arnaud M, et al. DPP-4 inhibitors and venous thromboembolism: an analysis of the WHO spontaneous reporting database. Lancet Diabetes Endocrinol 2020;8(05): 365-367
2 Lu W, Sun S, Wei Jet al.. Dipeptidyl peptidase-4 inhibitors and risk of venous thromboembolism: data mining of FDA adverse event reporting system. Int J Clin Pharm 2020;42(05):1364-1368

3 Higgins JP, Altman DG, Gøtzsche PC, et al; Cochrane Bias Methods Group; Cochrane Statistical Methods Group. The Cochrane Collaboration's tool for assessing risk of bias in randomised trials. BMJ 2011;343:d5928

4 Scirica BM, Bhatt DL, Braunwald E, et al; SAVOR-TIMI 53 Steering Committee and Investigators. Saxagliptin and cardiovascular outcomes in patients with type 2 diabetes mellitus. N Engl J Med 2013;369(14):1317-1326

5 Gantz I, Chen M, Suryawanshi S, et al. A randomized, placebocontrolled study of the cardiovascular safety of the once-weekly DPP-4 inhibitor omarigliptin in patients with type 2 diabetes mellitus. Cardiovasc Diabetol 2017;16(01):112

6 Green JB, Bethel MA, Armstrong PW, et al; TECOS Study Group. Effect of sitagliptin on cardiovascular outcomes in type 2 diabetes. N Engl J Med 2015;373(03):232-242

7 Rosenstock J, Perkovic V, Johansen OE, et al; CARMELINA Investigators. Effect of linagliptin vs placebo on major cardiovascular events in adults with type 2 diabetes and high cardiovascular and renal risk: the CARMELINA randomized clinical trial. JAMA 2019;321(01):69-79

8 White WB, Cannon CP, Heller SR, et al; EXAMINE Investigators. Alogliptin after acute coronary syndrome in patients with type 2 diabetes. N Engl J Med 2013;369(14):1327-1335

9 Arnaud M, Bégaud B, Thiessard F, et al. An automated system combining safety signal detection and prioritization from healthcare databases: a pilot study. Drug Saf 2018;41(04):377-387

10 Gupta AK, Verma AK, Kailashiya J, Singh SK, Kumar N. Sitagliptin: anti-platelet effect in diabetes and healthy volunteers. Platelets 2012;23(08):565-570

11 van Poppel PC, Netea MG, Smits P, Tack CJ. Vildagliptin improves endothelium-dependent vasodilatation in type 2 diabetes. Diabetes Care 2011;34(09):2072-2077 